\title{
Tongue Refolding in the Knotless Cyanobacterial
}

\section{Phytochrome All2699}

\author{
Qian-Zhao Xu, ${ }^{+,+}$Lukas Goett-Zink,,$^{\S}$ Wolfgang Gärtner, ${ }^{\dagger}$ Kai-Hong Zhao, *, \\ and Tilman Kottke $e^{*}$,
}

$\dagger$ Institute for Analytical Chemistry, University of Leipzig, Linnéstr. 3, 04109 Leipzig, Germany

$¥$ State Key Laboratory of Agricultural Microbiology, Huazhong Agricultural University, Wuhan 430070, China

$\S$ Physical and Biophysical Chemistry, Department of Chemistry, Bielefeld University, Universitaetsstr. 25, 33615 Bielefeld, Germany.

\section{Corresponding Authors}

* E-mail: tilman.kottke@uni-bielefeld.de (T.K.)

*Email: khzhao@163.com (K.-H.Z.). 
Table S1. Primers used for cloning gafl and gafl-gaf2 with wild-type and mutated sequences.

\begin{tabular}{|c|c|c|c|}
\hline Primer & $\mathrm{Se}$ & dence & $\mathrm{DNA}$ \\
\hline $\mathrm{P} 1$ & 51 & GCCATATGTCACCGACCGCTAAAC $3^{\prime}$ & gaf1 \\
\hline P2 & $5^{\prime}$ & GCCTCGAGATGACTTTGGGCGAT $33^{\prime}$ & $\operatorname{gaf1}$ \\
\hline P3 & $5^{\prime}$ & GTCATATGTCACCGACCGCTAAACC $3{ }^{\prime}$ & gaf1-gaf2 \\
\hline P 4 & $5^{\prime}$ & CACTCGAGTTCGTTAAAGGCTTGTACT & gaf1-gaf2 \\
\hline P5 & $5^{\prime}$ & GATGCCAAAAAGTCACAAGCTCA $33^{\prime}$ & R387P \\
\hline P 6 & $5^{\prime}$ & GGGCCATAAATTAAATGATACACG 3 ' & R387P \\
\hline P7 & $5^{\prime}$ & САтАААттТАТССАGATGGAAGCG & Y55H \\
\hline P8 & $5^{\prime}$ & AATCATCACTCGGTCAGTTCCCAAG & Y55H \\
\hline
\end{tabular}




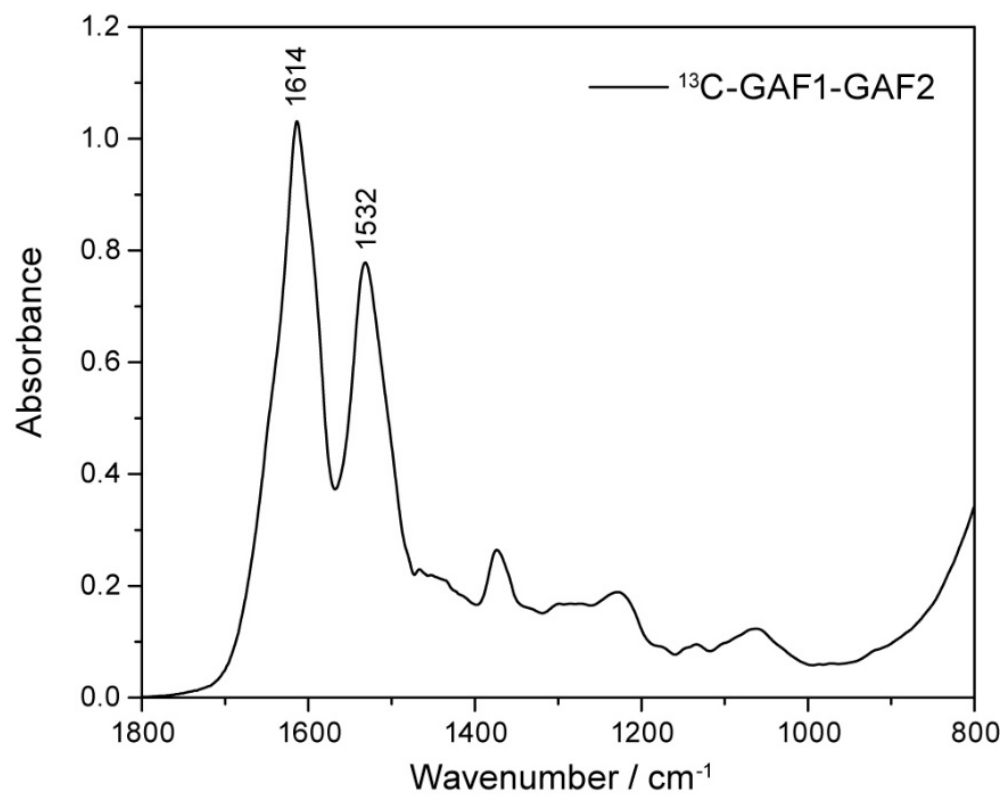

Figure S1. IR absorption spectrum of a dried sample of ${ }^{13} \mathrm{C}$-labeled GAF1-GAF2. The amide I band $\left(\mathrm{C}=\mathrm{O}\right.$ stretch of the backbone) is shifted from $\sim 1650 \mathrm{~cm}^{-1}$ to $1614 \mathrm{~cm}^{-1}$ by the ${ }^{13} \mathrm{C}$-labeling. Similarly, the amide II band is shifted from $\sim 1550 \mathrm{~cm}^{-1}$ to $1532 \mathrm{~cm}^{-1}$. The large extent of these shifts supports a complete labeling of the protein moiety with ${ }^{13} \mathrm{C}$. 


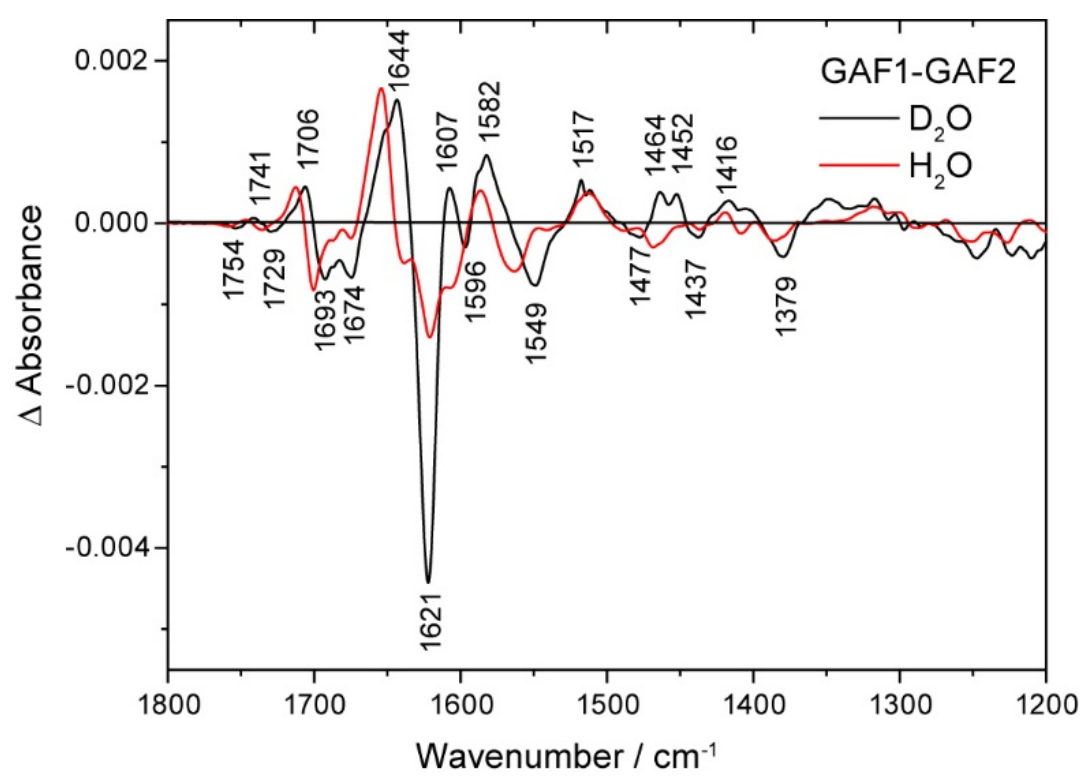

Figure S2. Light-induced FTIR difference spectrum of GAF1-GAF2 in $\mathrm{D}_{2} \mathrm{O}$ after illumination with red light at $632 \mathrm{~nm}$. The signals of tongue refolding shift from $1621(-) / 1654(+) \mathrm{cm}^{-1}$ to $1621(-) / 1644(+) \mathrm{cm}^{-1}$. Accordingly, the $\mathrm{H} / \mathrm{D}$ exchange in the $\alpha$-helix is much more pronounced than in the $\beta$-sheet. The signal increase of the latter is explained by the downshift of a compensating, positive contribution to $1607 \mathrm{~cm}^{-1}$ in $\mathrm{D}_{2} \mathrm{O}$. The signals of the D-ring carbonyl downshift from $1736(-) / 1712(+) \mathrm{cm}^{-1}$ to $1729(-) / 1706(+) \mathrm{cm}^{-1}$ upon H/D exchange as an indication for a moderate hydrogen bond strength in Pr and Pfr states. 


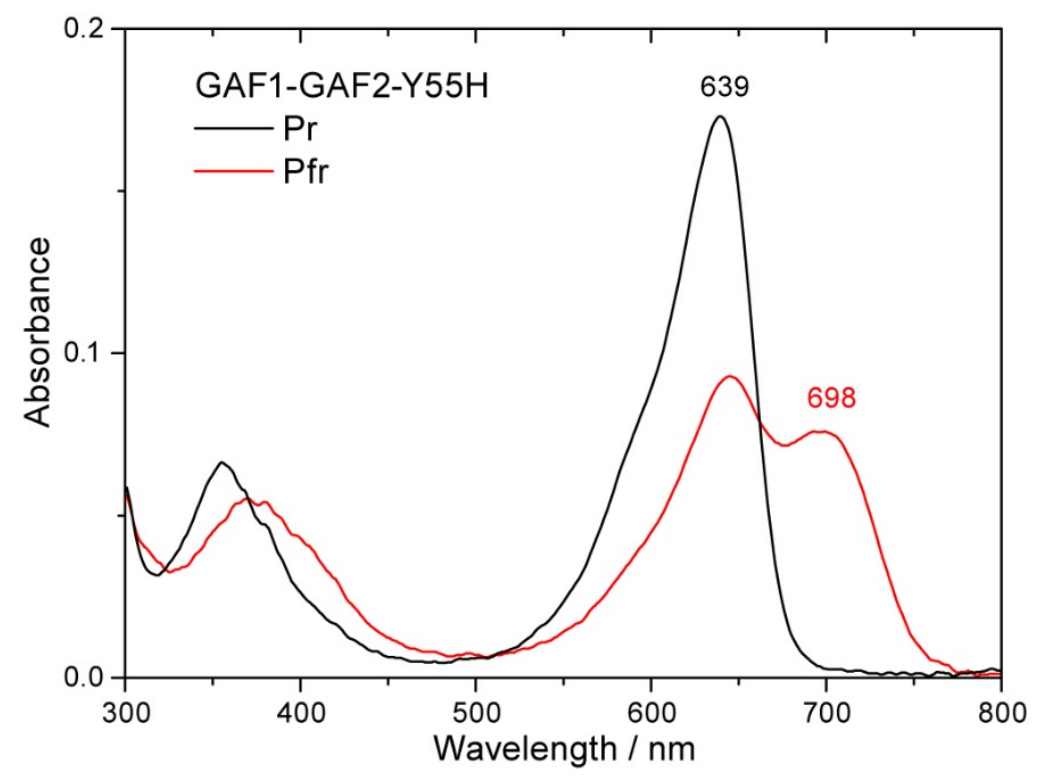

Figure S3. UV-vis spectra of the Pr and Pfr states of GAF1-GAF2-Y55H. The sample was photoconverted by illumination at $590 \mathrm{~nm}$. The maxima of absorbance are close to those of the wild-type GAF1-GAF2 with 638 and 705 nm, respectively. Accordingly, the exchange does not interfere with formation of the Pfr state. 